\title{
EDITORIAL
}

\section{NSIP, UIP, and the ABCs of idiopathic interstitial pneumonias}

\author{
J.L. Myers
}

Diffuse interstitial lung disease (ILD) is a general term encompassing a broad range of largely unrelated conditions which share the propensity to cause breathlessness and/or cough, while resulting in bilateral abnormal opacities of various types on conventional chest radiographs or computed tomography (CT) scans. The idiopathic interstitial pneumonias are a subset of diffuse ILDs characterized by an expansion of the interstitial compartment (i.e. that portion of the lung parenchyma sandwiched between the epithelial and endothelial basement membranes) by an infiltrate of inflammatory cells. The inflammatory infiltrate is sometimes accompanied by fibrosis, either in the form of abnormal collagen deposition or a proliferation of fibroblasts capable of collagen synthesis.

Our understanding of the idiopathic interstitial pneumonias has been plagued by our inability to clearly define the extent to which these represent distinct nosological entities as opposed to variations on the same fundamental theme. "Lumpers" would argue that all are appropriately covered under the venerable umbrellas of idiopathic pulmonary fibrosis (IPF), also referred to as cryptogenic fibrosing alveolitis (CFA), and that morphological separations are misguided attempts to segregate what are in fact highly related points on the same mysterious continuum of fibrosing lung disease. "Splitters" would respond that failure to control for histological subtypes has impeded our efforts to explain or predict the seemingly capricious behaviour of a family of similar but separable ILDs.

LIEBOw [1] pioneered the notion that morphological characteristics are useful in separating idiopathic interstitial pneumonias into clinically and histologically distinct groups (table 1). Liebow's categories of usual interstitial pneumonia (UIP) and desquamative interstitial pneumonia (DIP) have persisted as important histological groups, while bronchiolitis obliterans with classical interstitial pneumonia (BIP), lymphoid interstitial pneumonia (LIP) and giant cell interstitial pneumonia (GIP) have disappeared from more recent classification schemes (table 2) [2]. Liebow was careful to say that these were histological patterns rather than free-standing diagnostic entities, and that each could occur in a variety of clinical contexts. Regardless of the clinical context, however, he maintained that precise histological classification of interstitial pneumonias

Table 1. - Classification of idiopathic interstitial pneumonias in LIEBOW [1]

Usual interstitial pneumonia (UIP)

Desquamative interstitial pneumonia (DIP)

Bronchiolitis obliterans with interstitial pneumonia (BIP)

Lymphoid interstitial pneumonia (LIP)

Giant cell interstitial pneumonia (GIP)

Correspondence: J.L. Myers, Dept of Laboratory Medicine and Pathology, Mayo Clinic, 200 First St. SW, Rochester, MN 55905, USA. Fax: 1 5072841599 provides "clues both to aetiology and to pathogenesis and certainly to natural history and prognosis" [1]. In other words, although histological patterns are not free-standing diagnostic entities, each significantly limits the differential diagnosis in terms of potential aetiologies or clinical associations and each has specific implications concerning the likely treatment response and outcome. From a practical standpoint the majority of patients in whom open or thoracoscopically guided lung wedge biopsies show the histological patterns encompassed under the heading of idiopathic interstitial pneumonias are suspected of having IPF/CFA, and the biopsy is intended not only to confirm the suspicion of an interstitial pneumonia but also to exclude various IPF mimics such as sarcoidosis, hypersensitivity pneumonitis and pulmonary eosinophilic granuloma. It is in this very context that morphological classification has proven to be a powerful tool in predicting prognosis.

KatZENSTEIN and FIORELL [3] coined the term nonspecific interstitial pneumonia (NSIP) to formalize the long held suspicion that not all histologically documented int-erstitial pneumonias could be classified using the cate-gories prescribed by Liebow. NSIP was defined more in terms of what it is not rather than what it is. NSIP is defined as those chronic interstitial pneumonias that lack the heterogeneity and variegated pattern of UIP while also lacking the extensive accumulation of pigmented alveolar histiocytes that distinguish DIP. Sixteen per cent of the 64 original patients in KaTZENSTEIN and FioReLLI [3] had und-erlying collagen vascular disease of various sorts, while an equal number had potential organic antigen exposures that may have explained their lung disease. Five additional patients had histories suggesting antecedent acute lung injury, raising the possibility that NSIP may represent a resolving phase of diffuse alveolar damage (DAD) in some patients. A substantial number of patients remained, however, in whom NSIP was present in the context of susp-ected IPF/ CFA. Of the patients in whom follow-up was available, nearly half were alive and well, just under $40 \%$ had stable disease, and only approximately $10 \%$ had died. Interestingly, patients with fibrosis seemed to be at greater risk of dying than those with cellular, non-fibrotic lesions. KATZENSTEIN and FIORELLI [3] concluded that a pathological diagnosis of NSIP predicted greater likelihood of steroid responsiveness and a better outcome than classical UIP. BJORAKER et al. [4] subsequently provided additional evidence that there is a significant survival advantage in pa-tients with NSIP compared to patients with UIP.

Table 2. - Revised classification of idiopathic interstitial pneumonias [2]

Usual interstitial pneumonia (UIP)

Respiratory bronchiolitis interstitial lung disease (RBILD)/

desquamative interstitial pneumonia (DIP)

Acute interstitial pneumonia (AIP)

Nonspecific interstitial pneumonia (NSIP) 
In this issue of the journal, NAGAI et al. [5] report their experience with NSIP and contrast their findings with idiopathic bronchiolitis obliterans-organizing pneumonia (BOOP) and UIP. Patients with underlying or associated conditions, such as collagen vascular diseases or possible drug toxicity, were excluded. Thirty-one patients with NSIP formed the study set and were accrued from a variety of Japanese medical centres. NSIP patients were extensively reviewed at an international conference held in Kyoto in 1995 which included clinicians, radiologists, and pathologists. Patients with NSIP were further subdivided based on the presence or absence of fibrosis in the lung biopsy. The NSIP study group was compared to 34 patients with BOOP derived from a similar international conference convened in 1991, and 64 patients with UIP collected from the files of the Chest Disease Research Institute in Kyoto. The results confirm and expand the conclusions of Katzenstein, Bjoraker and others. Most of the features that distinguished NSIP from UIP also characterize the differences between BOOP and UIP, a finding that should not cause the reader to conclude that NSIP and BOOP are in fact the same entity. The most important clinical characteristics that distinguished NSIP from UIP were the lack of a strong predilection for occurring in males, a subacute rather than an insidious onset, associated fever in about a third of cases, and the relative lack of clubbing, which occurred in only $3(9.7 \%)$ patients with NSIP compared with 42 (65.6\%) patients with UIP. Crackles were common in both groups. Patients with NSIP had significantly higher carbon monoxide diffusing capacity of the lung values, but lower arterial oxygen tension values in the "fibrotic" (as opposed to "cellular") NSIP group. CT scans were less likely to show reticular/nodular opacities and honeycomb changes in patients with NSIP, while ground glass opacities and areas of consolidation were far more common. Bronchoalveolar lavage was also helpful in distinguishing NSIP from UIP in that a tendency toward increased cell recovery was accompanied by lymphocytosis and a decreased ratio of CD4:CD8 Tlymphocytes.

The most compelling distinction between NSIP and UIP was related to outcome: NSIP had a significantly better prognosis than UIP. Treatment was similar in both groups, although corticosteroids with or without immunosuppressants were used more frequently in patients with NSIP $(61.3 \%)$ than patients with UIP (46.9\%). Nearly $75 \%$ of patients with NSIP improved or recovered, a finding that characterized none of the patients with UIP. Five (16.1\%) patients with NSIP had progressive disease, including two who died either of progressive disease or complications of therapy, compared to a mortality rate of $76.7 \%$ (49 of 64 patients) for UIP. All of the patients with NSIP who suffered a more aggressive course were in the fibrotic subset. This group was interesting as honeycombing was present in either the lung biopsies (nine patients) or CT scans (two patients) of $11(73.3 \%)$ patients. In contrast, KATZENSTEIN and FIORELL [3] noted honeycomb change in $<10 \%$ of cases, including two of nine cases with a predominantly fibrotic pattern. The fibrotic changes in the fibrotic NSIP cases described in NAGAI et al. [5] were further characterized as having the same peripheral subpleural distribution as that typically seen in UIP, differing only in that they were more "temporally homogeneous". These observations at least raise the possibility that some patients with fibrotic NSIP may in fact have UIP which is not fully represented in the lung biopsies. The observations by S. Nagai and colleagues are an important addition to the growing body of evidence supporting the value of separating NSIP from classical UIP. It is no longer reasonable to consider IPF/CFA a distinct entity without limiting the definition to a specific morphological subset. Although historically these terms encompassed a morphologically heterogeneous group of patients, IPF/CFA should now be either restricted to those patients with classical UIP or abandoned altogether. UIP, in turn, is a specific form of fibrosing interstitial pneumonia distinguished by a highly variegated appearance resulting from a temporally heterogeneous pattern of ongoing lung injury. Defined in this fashion, IPF/CFA emerges as a lesion with a uniformly poor prognosis that rarely if ever resolves and nearly always progresses despite current immunosuppressive regimens. There is little doubt that NSIP explains many historical observations regarding a "good prognosis" subset of IPF/CFA, an oxymoronic notion that can now be laid to rest. It also seems likely that NSIP explains historical observations regarding the value of a "cellular" biopsy in predicting steroid responsiveness, another notion that may not apply to classical UIP. Whether we can go one step further and conclude that NSIP is, paradoxically, a specific clinicopathological entity will remain controversial for the moment.

Critics will continue to argue that NSIP is merely early UIP, that NSIP is not a disease, and that NSIP is a poor choice of terms. There is little evidence from the published experience to suggest that NSIP is an early form of UIP. Although problematic cases will continue to blur the distinction between "fibrotic" NSIP and classical UIP, difficult diagnosis cases should not be used as anecdotal evidence to discredit a notion built on broader experience. NSIP, like the other morphological patterns of idiopathic interstitial pneumonia, is not a disease until viewed in the appropriate and narrowly defined clinical context. As to the choice of terms, there is little precedent for thinking that altering the label will have any meaningful impact on our ability to understand the postulate. These minor issues must not distract us from the fundamental principle that should inform current thinking regarding idiopathic interstitial pneumonias: however IPF/CFA may have been used in the past, these terms should now be restricted to those patients with UIP, a specific category of fibrosing ILDs associated with a poor prognosis. Our chief mission in practice and in science is to identify those things that are something else, particularly when it has implications for patient management and outcome. Indeed, the predictions of LEBBow [1] have been reinvigorated by renewed interest in the value of histological patterns in providing important clues, "both to aetiology and to pathogenesis and certainly to natural history and prognosis".

\section{References}

1. Liebow A. Definition and classification of interstitial pneumonias in human pathology. Prog Respir Res 1975; 8: 1-33

2. Katzenstein A-L, Myers J. Idiopathic pulmonary fibrosis: clinical relevance of pathologic classification. $A m J$ Respir Crit Care Med 1998; 157: 1301-1315.

3. Katzenstein A-L, Fiorelli R. Nonspecific interstitial pneumonia/fibrosis: histologic features and clinical significance. Am J Surg Pathol 1994; 18: 136-147.

4. Bjoraker J, Ryu J, Edwin M, et al. Prognostic significance of histopathological subsets in idiopathic pulmonary fibrosis. Am J Respir Crit Care Med 1998; 157: 199-203.

5. Nagai $\mathrm{S}$, Kitaichi $\mathrm{M}$, Itoh $\mathrm{H}$, et al. Idiopathic nonspecific interstitial pneumonia/fibrosis: comparison with idiopathic pulmonary fibrosis and bronchiolitis obliterans organizing pneumonia. Eur Respir J 1998; 12: 1010-1019. 